\title{
Anti-vinculin autoantibodies in systemic sclerosis: a step toward a novel biomarker?
}

\author{
Brittany L. Adler ${ }^{1}$ (D) $\cdot$ Zsuzsanna McMahan $^{1}$
}

Received: 12 November 2020 / Revised: 27 November 2020 / Accepted: 1 December 2020 / Published online: 7 January 2021

(C) International League of Associations for Rheumatology (ILAR) 2021

Systemic sclerosis ( $\mathrm{SSc}$ ) is an autoimmune systemic vasculopathy that leads to wide-spread organ fibrosis. Gastrointestinal (GI) involvement in SSc is common and often results in debilitating symptoms that can lead to malnutrition and in severe cases dependence on total parenteral nutrition (TPN). There are multiple causes of GI symptoms in SSc, including dysfunction of the lower esophageal sphincter which can lead to chronic gastrointestinal reflux disease (GERD) and strictures, GI dysbiosis [1] including small intestinal bowel overgrowth (SIBO), food intolerance (e.g., fructose, lactose) [2, 3], and dysmotility of the GI tract (i.e., esophagus, stomach, small and large bowel). Our understanding of GI dysbiosis in medicine, and particularly in SSc, is still evolving, and the relationship between transit abnormalities and dysbiosis is still poorly understood. While gastric emptying tests are widely available, other more specialized imaging tests to evaluate for dysmotility throughout the GI tract are limited in availability and not well-studied in SSc. Serum biomarkers to diagnose and monitor GI disease in SSc are lacking, especially biomarkers that can reliably detect early GI disease activity. In addition, questions remain about how to identify subgroups at high risk for GI progression and whether the early application of promotility agents or immunomodulation prior to the development of progressive smooth muscle atrophy and GI fibrosis is beneficial for such patients.

It is well-known that autoantibodies in SSc predict clinical phenotype [4]. Several studies have implicated anti-neuronal autoantibodies in SSc GI dysmotility, which are thought to reflect an immune response directed against the enteric nervous system. Autoantibodies targeting the myenteric plexus

Brittany L. Adler

Brittany.adler@jhmi.edu

1 Department of Medicine, Division of Rheumatology, Johns Hopkins University, Baltimore, MD 21224, USA are present in a subset of SSc patients [5], as well as muscarinic-3 acetylcholine receptor autoantibodies (antiM3R) and anti-ganglionic acetylcholine receptor autoantibodies (anti-gAChR) which associate with severe GI involvement $[6,7]$. However, these autoantibodies are not present in all SSc patients with GI disease, and so other autoantibodies likely remain to be discovered.

Dr. Suliman and colleagues identify autoantibodies targeting the protein vinculin in a SSc cohort that associate with GI symptom severity. Vinculin is a cytoplasmic protein that binds to actin and is involved in cell adhesion. The authors demonstrate that anti-vinculin autoantibody levels are higher and these autoantibodies are more frequently identified in a SSc cohort enriched with GI disease compared with a healthy control population. Anti-vinculin autoantibody levels also associate with the severity of GI symptoms as measured by the GI-visual analogue score (GI-VAS).

The presence of anti-vinculin autoantibodies in SSc is consistent with prior studies which have implicated vinculin as an important regulator of the enteric nervous system. Vinculin is notably involved in neuronal migration and axon growth, and vinculin deletion from mouse neocortical neurons results in attenuation of axon growth in vivo [8]. One study found an inverse association between anti-vinculin autoantibody titers and the density of interstitial cells of Cajal (ICC) in the myenteric plexus of human stomach from patients with gastric cancer [9]. The primary function of ICC is to serve as the pacemaker for gut motility by mediating neurotransmission between the autonomic nervous system and smooth muscle cells [10], and so the low density of ICC among patients with anti-vinculin autoantibodies suggests that gastric ICC may be affected by this aberrant immune response targeting vinculin. Some patients with SSc have reduced esophageal ICC [11], although the presence of anti-vinculin autoantibodies among these patients remains unknown.

Autoantibodies targeting vinculin were first discovered in irritable bowel syndrome (IBS) [12], a GI dysmotility syndrome that is thought to be in part immunologically mediated 
and potentially triggered by gastroenteritis [13]. Anti-vinculin autoantibodies are specific for IBS compared with inflammatory bowel disease (IBD) [14]. However, the presence of antivinculin autoantibodies in SSc GI disease suggests that these autoantibodies are not highly specific for IBS, and further studies are needed to determine if they are present in other autoimmune GI dysmotility syndromes as well. This finding also raises questions about the pathogenesis of anti-vinculin autoantibodies in SSc that warrants further exploration. Data in IBS suggest that the toxin produced by bacteria that commonly causes gastroenteritis and subsequent IBS may result in autoantibodies that cross-react with vinculin and impair gut motility. This mechanism seems less plausible in SSc, which typically presents with involvement of other organ systems such as Raynaud's and is not known to temporally associate with gastroenteritis, although anecdotally a subset of patients do attribute their onset of SSc to a preceding flu-like or GI illness. More work needs to be done to understand if antivinculin autoantibodies are directly pathogenic in SSc or whether they are simply a marker of GI dysfunction (Fig. 1).

What, if anything, are the clinical implications of the discovery of anti-vinculin autoantibodies for the diagnosis and management of SSc GI disease? Given that motility testing used to diagnose SSc GI disease is time-consuming and is often only offered at specialized centers, a non-invasive serum biomarker would be a welcome addition to the field.
However, much more work is needed before anti-vinculin autoantibodies are ready for the clinical setting. In this study the authors analyzed the relationship between anti-vinculin autoantibodies and self-reported GI symptoms, which are a poor outcome measure as GI symptoms are unable to differentiate between the multiple GI pathologies that afflict SSc patients (e.g., dysmotility, dysbiosis, food intolerances, and anatomical abnormalities (e.g. esophageal strictures)). Antivinculin autoantibodies may have a role in the clinical setting if studies can demonstrate that these autoantibodies reliably distinguish between dysmotility and other GI pathology in SSc with similar sensitivity and specificity compared with standard imaging modalities. Furthermore, self-reported GI symptoms do not help localize the area of GI pathology. For example, nausea can result from GERD, gastroparesis, or small or large bowel dysmotility. Future studies using more specialized tests such as whole-gut transit scintigraphy or motility capsules $[15,16]$ are needed to determine if anti-vinculin autoantibodies can localize the area of GI dysmotility and therefore help guide treatment decisions. It will also be clinically important to understand whether anti-vinculin autoantibodies are present in early SSc GI disease or whether they develop as a late SSc GI manifestation. Although controversial, it is possible that early GI disease may be reversible with immunomodulation or the application of promotility agents. And lastly, it will be interesting to see if anti-vinculin
Fig. 1 Model for pathogenesis of irritable bowel syndrome proposed by Mark Pimentel et al., Dig Dis Sci (2015) 60:1195-1205 (PMID: 25424202). Vinculin autoantibodies may also be important in the pathogenesis of scleroderma gastrointestinal dysfunction

\section{Proposed Model for Pathogenesis of Irritable Bowel Syndrome}

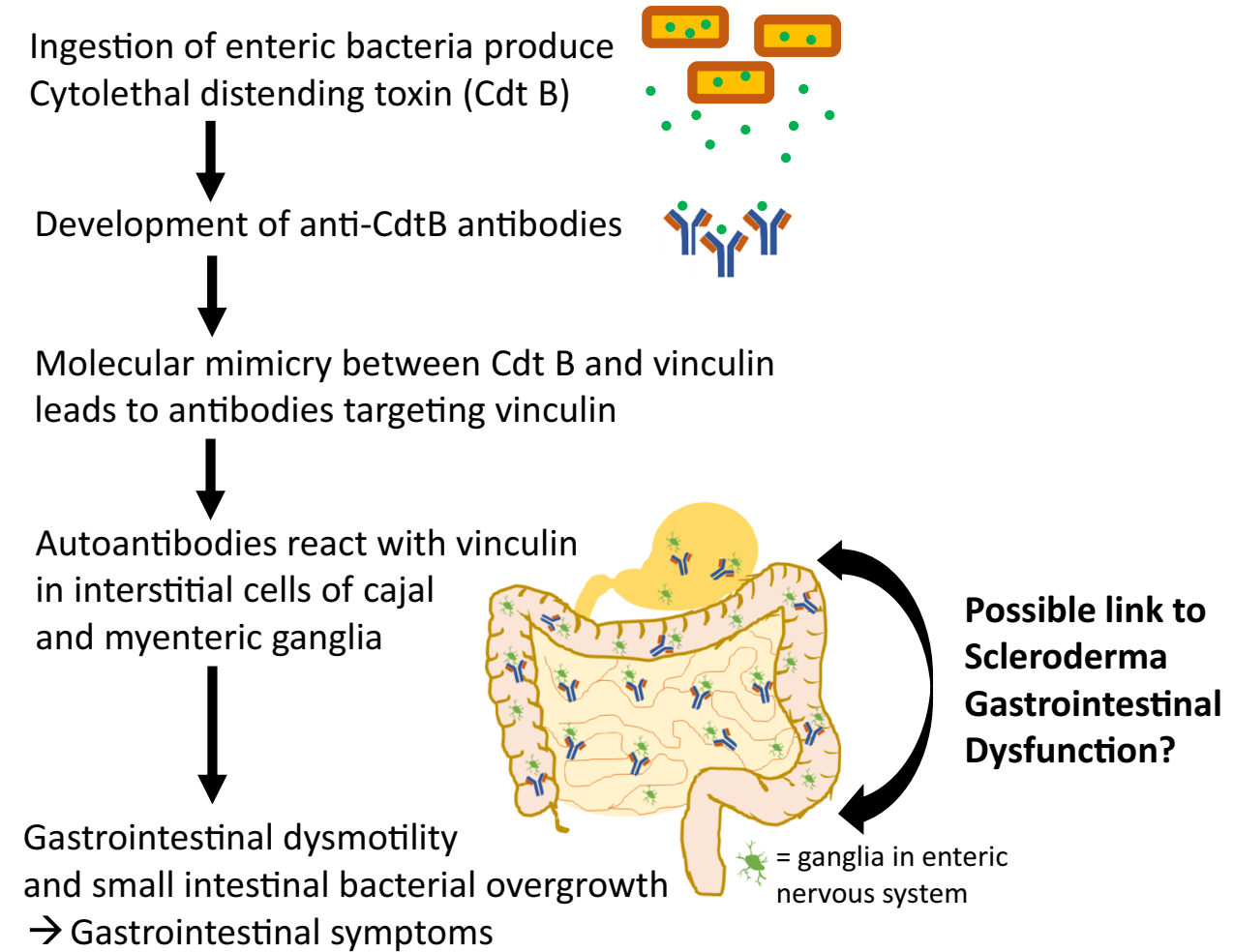


autoantibodies have a role in non-invasively monitoring the progression of the disease, thus serving as a marker of GI disease activity. Although important, this is challenging to study given that histopathology is necessary to distinguish between GI disease activity and smooth muscle atrophy/ chronic damage.

The authors also show a trend toward more pulmonary arterial hypertension (PAH) among patients with antivinculin autoantibodies in the vascular-enriched group. This is an interesting finding given that a subset of SSc patients with pulmonary hypertension have anti-endothelial cell autoantibodies and vinculin has been previously shown to be a target antigen of these autoantibodies [17]. However, future studies are needed with a larger patient population to confirm this association.

In summary, Dr. Suliman and colleagues propose a novel autoantibody targeting vinculin in SSc that associates with severe GI symptoms. This finding provides possible insights into the mechanisms underlying SSc GI dysfunction and shows some interesting parallels with other immune-based dysmotility syndromes such as IBS. Further studies are needed to determine the pathogenic relevance of these autoantibodies in SSc and determine whether they have utility in the clinical setting for the diagnosis and management of SSc.

Funding Scleroderma Research Foundation (BA and ZM) and National Institute of Arthritis and Musculoskeletal and Skin Diseases (ZM, 1K23AR071473).

\section{Compliance with ethical standards}

Disclaimer This Editorial represents the opinions of the authors and do not reflect those of the National Institutes of Health (NIH) or the National Institute of Arthritis Musculoskeletal and Skin Diseases (NIAMS).

Disclosures None.

\section{References}

1. Volkmann ER, Hoffmann-Vold AM, Chang YL, Jacobs JP, Tillisch K, Mayer EA, Clements PJ, Hov JR, Kummen M, Midtvedt Ø, Lagishetty V, Chang L, Labus JS, Molberg Ø, Braun $\mathrm{J}$ (2017) Systemic sclerosis is associated with specific alterations in gastrointestinal microbiota in two independent cohorts. BMJ Open Gastroenterol 4:e000134. https://doi.org/10.1136/bmjgast-2017000134

2. Marie I, Leroi AM, Gourcerol G, Levesque H, Ménard JF, Ducrotte P (2015) Fructose malabsorption in systemic sclerosis. Med (United States) 94:e1601. https://doi.org/10.1097/MD.0000000000001601

3. Marie I, Leroi A-M, Gourcerol G, Levesque H, Menard JF, Ducrotte P (2016) Lactose malabsorption in systemic sclerosis. Aliment Pharmacol Ther 44:1123-1133. https://doi.org/10.1111/ apt. 13810

4. Ho KT, Reveille JD (2003) The clinical relevance of autoantibodies in scleroderma. Arthritis Res Ther 5:80-93
5. Howe S, Eaker EY, Sallustio JE, Peebles C, Tan EM, Williams RC $\mathrm{Jr}$ (1994) Antimyenteric neuronal antibodies in scleroderma. J Clin Invest 94:761-770. https://doi.org/10.1172/JCI1 17395

6. Kawaguchi Y, Nakamura Y, Matsumoto I, Nishimagi E, Satoh T, Kuwana M, Sumida T, Hara M (2009) Muscarinic-3 acetylcholine receptor autoantibody in patients with systemic sclerosis: contribution to severe gastrointestinal tract dysmotility. Ann Rheum Dis 68 : 710-714. https://doi.org/10.1136/ard.2008.096545

7. Nakane S, Umeda M, Kawashiri SY, Mukaino A, Ichinose K, Higuchi O, Maeda Y, Nakamura H, Matsuo H, Kawakami A (2020) Detecting gastrointestinal manifestations in patients with systemic sclerosis using anti-gAChR antibodies. Arthritis Res Ther 22:1-10. https://doi.org/10.1186/s13075-020-2128-z

8. Mandal P, Belapurkar V, Nair D, Ramanan N (2020) Vinculin mediated axon growth requires interaction with actin but not talin. bioRxiv 2020.06.29.177758. https://doi.org/10.1101/2020.06.29. 177758

9. Kim JH, Nam SJ, Park SC, Lee SH, Kim TS, Lee M, Park JM, Choi DH, Kang CD, Lee SJ, Ryu YJ, Lee K, Park SY (2020) Association between interstitial cells of Cajal and anti-vinculin antibody in human stomach. Korean J Physiol Pharmacol 24:185-191. https://doi. org/10.4196/kjpp.2020.24.2.185

10. Sanders KM (1996) A case for interstitial cells of Cajal as pacemakers and mediators of neurotransmission in the gastrointestinal tract. Gastroenterology 111:492-515. https://doi.org/10.1053/gast. 1996.v111.pm8690216

11. Roberts CGP, Hummers LK, Ravich WJ, Wigley FM, Hutchins GM (2006) A case-control study of the pathology of oesophageal disease in systemic sclerosis (scleroderma). Gut 55:1697-1703. https://doi.org/10.1136/gut.2005.086074

12. Rezaie A, Park SC, Morales W, Marsh E, Lembo A, Kim JH, Weitsman S, Chua KS, Barlow GM, Pimentel M (2017) Assessment of Anti-vinculin and anti-cytolethal distending toxin $\mathrm{B}$ antibodies in subtypes of irritable bowel syndrome. Dig Dis Sci 62:1480-1485. https://doi.org/10.1007/s10620-017-4585-z

13. Morales W, Triantafyllou K, Parodi G, Weitsman S, Park SC, Rezaie A, Pichetshote N, Lin E, Pimentel M (2020) Immunization with cytolethal distending toxin B produces autoantibodies to vinculin and small bowel bacterial changes in a rat model of postinfectious irritable bowel syndrome. Neurogastroenterol Motil 32:e13875. https://doi.org/10.1111/nmo. 13875

14. Pimentel M, Morales W, Rezaie A, Marsh E, Lembo A, Mirocha J, Leffler DA, Marsh Z, Weitsman S, Chua KS, Barlow GM, Bortey E, Forbes W, Yu A, Chang C (2015) Development and validation of a biomarker for diarrhea-predominant irritable bowel syndrome in human subjects. PLoS One 10:e0126438. https://doi.org/10. 1371/journal.pone. 0126438

15. Bonapace ES, Maurer AH, Davidoff S, Krevsky B, Fisher RS, Parkman HP (2000) Whole gut transit scintigraphy in the clinical evaluation of patients with upper and lower gastrointestinal symptoms. Am J Gastroenterol 95:2838-2847. https://doi.org/10.1111/j. 1572-0241.2000.03195.x

16. Saad RJ, Hasler WL (2011) A technical review and clinical assessment of the wireless motility capsule. Gastroenterol Hepatol 7:795804

17. Dib H, Tamby MC, Bussone G, Regent A, Berezné A, Lafine C, Broussard C, Simonneau G, Guillevin L, Witko-Sarsat V, Humbert M, Mouthon L (2012) Targets of anti-endothelial cell antibodies in pulmonary hypertension and scleroderma. Eur Respir J 39:14051414. https://doi.org/10.1183/09031936.00181410

Publisher's note Springer Nature remains neutral with regard to jurisdictional claims in published maps and institutional affiliations. 